\title{
Effect of Pongamia pinnata flowers on blood glucose and oxidative stress in alloxan induced diabetic rats
}

Diabetes mellitus is a major metabolic syndrome characterized by derangement in carbohydrate metabolism associated with defect in insulin secretion or action. Alloxan is widely used to induce diabetes mellitus in experimental animals, owing to its ability to destroy the B-cells of pancreas possibly by generating excess reactive oxygen species. ${ }^{[1]}$ Free radical-mediated biomembrane lipid peroxidation has been implicated in the pathogenesis of many pathological conditions including diabetes mellitus and its complications. Overproduction of lipid peroxidation by-products and insufficient antioxidant potential have been reported in both experimental and human diabetes mellitus. ${ }^{[2]}$

Medicinal plants and their bioactive constituents are used for the treatment of diabetes mellitus throughout the world, especially in countries where access to the conventional treatment of diabetes mellitus is inadequate. A few side effects associated with the use of insulin (hypoglycemia) and oral hypoglycemic agents prompted us to search new bioactive principles from antidiabetic plants used in traditional medicine. Although several medicinal plants have gained importance for the treatment of diabetes mellitus, many remain to be scientifically investigated. Pongamia pinnata (Linn) Pierre is a medium-sized glabrous tree popularly known as Karanja in Hindi, Indian beech in English, and Pongam in Tamil. $P$. pinnata is an important medicinal plant chiefly found in tidal forests of India and has been largely used in the traditional Indian system of medicine (ayurveda) for bronchitis, whooping cough, rheumatic arthritis, and diabetes. ${ }^{[3 \mid}$ Despite its prominence in ayurvedic medicine, there is a dearth of scientific data on its antihyperglycemic and antilipidperoxidative effects in diabetes mellitus. Thus, the present study was focused on the antihyperglycemic and antilipidperoxidative activities of aqueous extract of $P$. pinnata flowers (PpFAet) in alloxan-induced diabetic rats.

Albino Wistar male rats (7-8 week old, weighing 150-200 g) were used in the present study and housed in the Central Animal House with $12 \mathrm{~h}$ light/dark cycle. Standard pellet (Mysore Snack Feed Ltd, Mysore, India) was used as a basal diet during the experiment. The control and experimental animals were provided food and water ad libitum. The Institutional Animal Ethics Committee of the Annamalai University, Annamalai Nagar, India, approved the experimental design. A total of 30 rats were divided into 5 groups of 6 each and treated as follows.

Group I-control (2 $\mathrm{ml}$ distilled water, orally).

Group II-diabetic control (alloxan, $150 \mathrm{mg} / \mathrm{kg}$, i.p).

Group III—diabetic + PpFAet (300 mg/kg, orally).

Group IV—diabetic+ glibenclamide $(600 \mu \mathrm{g} / \mathrm{kg}$, orally).

Group V-PpFAet + distilled water $(300 \mathrm{mg} / \mathrm{kg}$, orally).
The diabetic condition was assessed by determining the blood glucose concentration 3 and 5 days after alloxan treatment. The rats with blood glucose level above $260 \mathrm{mg} / \mathrm{dl}$ and urinary sugar $(+++)$ were selected for study (Groups II-IV). The dose (300 mg/kg, orally) was standardized after a pilot study with different doses of the PpFAet extract to assess the antihyperglycemic and antilipidperoxidative effects in alloxan-induced diabetic rats. After the experimental period, all animals were sacrificed by cervical dislocation and biochemical studies conducted in blood, plasma, and liver samples using colorimetric methods. Plasma insulin was assayed by ELISA method using Boehinger Mannheim Gmbh kit. The data are expressed as mean \pm SD. Statistical comparisons were performed by one-way avova followed by Dunnett's test. The results were considered statistically significant if $\mathrm{P} \leq 0.05$.

A significant decrease in the level of blood glucose and glucose-6-phosphatase activity and a significant increase in the plasma insulin level and hexokinase activity were noted at the end of the experimental period in diabetic rats treated with PpFAet (300 mg/kg, orally). PpFAet also significantly reduced the thiobarbituric acid-reactive substances (TBARS) level and enhanced the antioxidants status in induced diabetic rats after 45 days of treatment. PpFAet showed antihyperglycemic and antilipidperoxidative effects in a manner similar to that of the reference drug glibenclamide in alloxan-induced diabetic rats. [Table 1]

Diabetes mellitus is a chronic metabolic disorder characterized by hyperglycemia associated with several other factors including dyslipidemia, which are involved in the development of micro- and macrovascular complications. In the present study, the orally administered PpFAet (300 mg/ orally) to diabetic rats showed a significant antihyperglycemic activity as well as significantly increased the plasma insulin level. The extract also normalized the activities of glucose-6phosphatase and hexokinase in diabetic rats. Any defect in insulin and glucagon secretion brings about changes in glucose homeostasis and activities of key enzymes of glucose metabolism. Decreased hexokinase activity and increased glucose-6-phosphatase activity in diabetic rats is probably owing to insulin deficiency. Enhanced hexokinase activity and decreased glucose-6-phosphatase activity in PpFAet-treated diabetic rats, therefore, suggest its stimulatory effects on insulin secretion from remnant pancreatic B-cells, which in turn promotes greater utilization of blood glucose by the liver, muscle, and adipose tissues of diabetic rats.

Lipid peroxide-mediated tissue damage has been demonstrated in diabetes mellitus. Several studies indicated that the alloxan-induced diabetes mellitus involves the 


\section{R. Punitha, K. Vasudevan, ${ }^{\star}$ S. Manoharan}

Departments of Biochemistry and Zoology*, Faculty of Science, Annamalai University, Annamalai Nagar 608002, Tamil Nadu, India. E-mail: manshisak@yahoo.com

\section{References}

1. Szkuldeshi T. The mechanism of alloxan and streptozotocin action in B-cells of the rat pancreas. Physiol Res 2001;50:53646.

2. Vander Jagt DJ, Harrison JM, Ratliff DM, Hunsaker LA, Vander Jagt DL. Oxidative stress indices in IDDM subjects with and without long-term diabetic complications. Clin Biochem 2001;34:265-70.

3. Kritikar KR, Basu BD. Indian medicinal plants. $2^{\text {nd }}$ ed. Vol. 1, Allahabad, India; Lalit Mohan Basu; 1956. p. 830-2.

4. Hunnt JV, Smith CCT, Wolff SP. Auto oxidative glycosylation and possible involvement of peroxides and free radicals in LDL modification by glucose. Diabetes 1999;39:1420-4.

5. Felliet-Coudray C, Rock E, Coudray C, Grzelkowska K, Azais-Braesco V, Dardevet D. Lipid peroxidation and antioxidants status in experimental diabetes. Clin Chim Acta 1999;284:31-43.

6. Ananthan R, Latha M, Pari L, Baskar C, Narmatha V. Modulatory effects of Gymnema montanum leaf extract on alloxan induced oxidative stress in Wistar rats. Nutrition 2004;20:2805. 